\title{
Investigation of a slip joint connection between the monopile and tower of an offshore wind turbine
}

\author{
M.L.A. Segeren \\ Offshore Wind Group \\ Department of Offshore Engineering \\ Technical University of Delft \\ Email: m.I.a.segeren@tudelft.nl
}

\author{
E. Lourens \\ Offshore Wind Group \\ Department of Offshore Engineering \\ Technical University of Delft \\ Email: e.lourens@tudelft.nl
}

\section{Abstract}

To circumvent current industry problems related to the settling of grouted connections, a steel-to-steel or slip joint connection is proposed for fitting a transition piece onto an installed monopile foundation. In the first part of this contribution, a simplified dynamic analysis of the installation of such a joint is considered. Assuming velocity dependent Coulomb friction, slip-stick equations of motion are derived for a simplified 1-D model of the joint, and the slip distance under self weight and resulting overlap lengths are calculated for different initial cone angles and friction coefficients. It is concluded that even for small initial cone angles, small angle differences between the top and bottom cone, and low friction coefficients, the tangential displacement caused by the self weight is insufficient to reach the desired contact overlap.

In the second part of this contribution, the static capacities (axial and bending) of the joint in the in-place situation are determined by means of a FE model. Contact analyses are performed based on the ideal situation in which the two parts of the joint is initially in full contact. Of the parameters varied in these analyses, small cone angles and large overlaps are identified as most conducive to a succesfull transferral of the loads from the transition piece to the monopile. Given the uncertainty on the friction coefficient, it is then also recommended to use a cone angle of $1^{\circ}$ and preferably an overlap $>1.5 D$.

Keywords: Support structure, Monopile, Slip joint, Alternative transition piece

\section{Introduction}

It is current industry practice to use a grouted connection for fitting a transition piece onto an installed monopile foundation. In 2008, however, it was observed that the straight grouted connections at the offshore wind farm Egmond aan Zee (OWEZ) off the Dutch North Sea coast were settling. Subsequent surveys on other wind farms revealed that this was the case for grouted connections in most of the wind farms in the North Sea. The observed subsiding of the transition piece with respect to the pile has led to a review of the recommended design procedures for large diameter grouted connections. The review was performed in the framework of a joint industry project[1] and resulted in a revision of the Det Norske Veritas offshore standard for the design of wind turbine structures [2]. The revision now recommends that axial loads should be transferred in an alternative way, where one of the proposed solutions is that the pile should make a small angle with the vertical to prevent slippage. Complicating factors that were identified during the investigation include abrasive wear of the sliding contact surfaces between the steel and grout, as well as possible progressive grout crushing or cracking under reversed dynamic loading.

To circumvent these problems of the grouted connection a steel-to-steel connection, or slip joint, is proposed. A slip joint consists of two conical sections, one attached to the top of the foundation pile and the other to the bottom of the transition piece. The dimensions of the two sections are chosen so as to have one fit closely inside the other in the same way as two inverted cups. In figure 1. An onshore wind turbine located at Scheveningen on the Dutch coast was designed and installed with a slip joint connection and has already been in operation since 1995 [3]. Surveys carried out on this onshore turbine in 2001 and 2010 revealed no signs of 
deterioration of the joint. If the connection can also be used in offshore wind farms, it would remove all the current problems related to the grout. Other advantages include a saving on risk and grout costs and a possible acceleration of the installation process. For offshore applications, however, the far larger scales and harsher environmental conditions necessitate further research.

In this contribution two different aspects of the development of such a joint for offshore applications are considered, namely a) a simplified dynamic analysis of its installation under its own weight and b) a determination of its static capacities (axial and bending) in the in-place situation by means of an FE model.
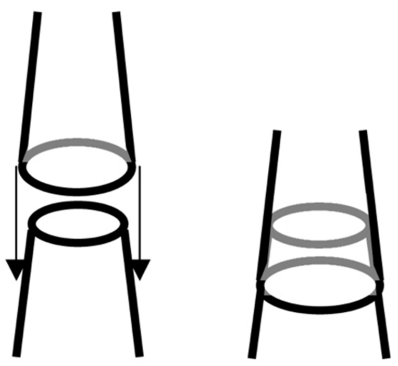

Figure 1: Slip joint concept

\section{Dynamic analysis of the installa- tion}

As an initial step towards dynamic modeling of the axisymmetric installation procedure, the two conical cylinders are represented as rigid beams as shown in figure 2. The geometrical stiffness of the top cone in the radial direction is represented by linear springs, whilst the bottom cone is assumed fixed. The contact stiffness between the bodies in normal and tangential direction are represented by two linear springs. In figure 3 the 1-D model of the slipjoint with rigid beams and contact stiffnesses is shown.

The radial stiffness $K_{r r}$ is a function of the outer radius $R_{U c}$ of the upper cone, the wall thickness $t_{w}$, the elasticity modulus $E$ and the Poisson ratio $\nu$. Using linearized theory of elasticity for 2-D problems with cylindrical coordinates [4], $K_{r r}$ can be derived as:

$$
K_{r r}=\frac{E \cdot\left({R_{i}}^{2}-R_{o}{ }^{2}\right)}{R_{i} \cdot(1+\nu) \cdot\left(-{R_{i}}^{2}+2{R_{i}}^{2} \nu-{R_{o}}^{2}\right)}
$$

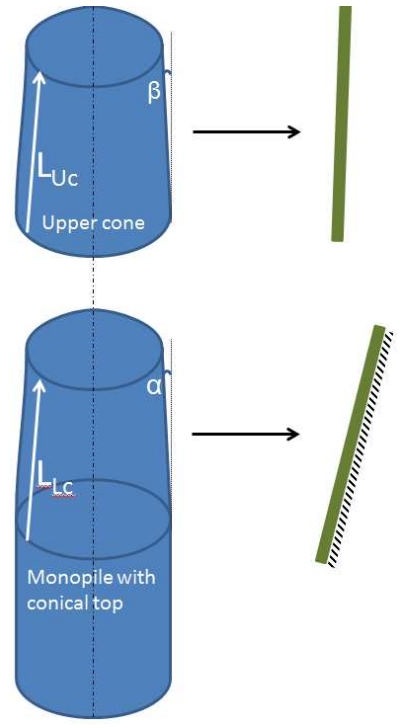

Figure 2: Transformation to 1-D

Note that the conical shape of the cylinder will introduce a stiffness in radial direction that changes over the height of the cylinder. For the mass-spring representation used to define the equation of motions in the next section, an equivalent spring stiffness will be used, obtained by integrating the radial stiffness function over the height of the upper cone.

The contact stiffnesses $K_{\tau}$ and $K_{N}$ in respectively tangential and normal direction can be derived with circular conical shell theory [8]. These stiffnesses represent the resistance of the conical shaped cylinder against a circumferential line load in either tangential or normal direction. The stiffnesses are dependent on the cone angle $\alpha$, the radius of the top of the lower cone $R_{L C}$, the wall thickness $t_{w}$, the elasticity modulus, the Poisson ratio, the length of the cone and the boundary conditions at the ends of the conical section.

\subsection{Slip-stick equations of motion}

The upper cone can respond to the gravitational load in either one of 2 ways. It can either stick to a given position, represented by $x_{\tau}$ in each slip-stick cycle, or it can start slipping/moving if the force in tangential direction is larger than the maximum friction force. Drawing on similar systems presented and discussed by Den Hartog [6], Popp [7] and Hong et al [5], a slip-stick system with a position and velocity dependent Coulomb friction force is introduced. In the following, the equations of 


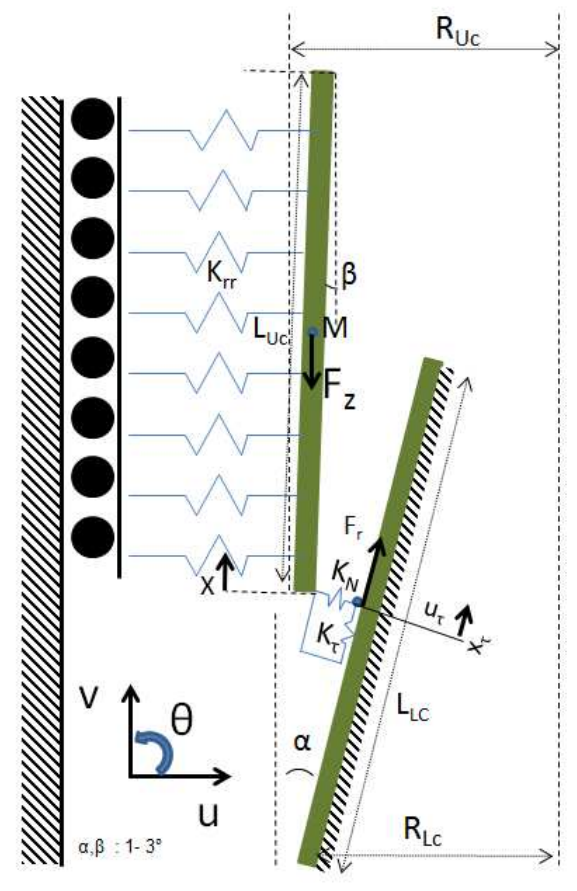

Figure 3: The 1-D model

motion are derived for the 1-D model of figure 3 represented using the mass-spring system with coulomb friction as illustrated in figure 4 .

The Coulomb friction force can be calculated as:

$$
F_{r}=\left\{\begin{array}{lll}
\mu_{d} N & \text { if } & \dot{u}_{\tau}>0 \\
\in\left[-\mu_{s} N, \mu_{s} N\right] & \text { if } & \dot{u}_{\tau}=0 \\
-\mu_{d} N & \text { if } & \dot{u}_{\tau}<0
\end{array}\right.
$$

Where $\mu_{d}, \mu_{s}$ and $\mathrm{N}$ are the dynamic and static friction coefficients and the normal force respectively. The friction coefficients $\mu_{d}$ and $\mu_{s}$ are assigned values of 0.5 and 0.4 , respectively.

This friction force shows that motion of the slipjoint will be piecewise and dependent on the tangential velocity. The friction coefficient $\mu$ that is used in this model is discontinuous and is taken as:

$$
\mu=\left\{\begin{array}{llll}
\mu_{s} & =0.5 & \text { if } & \dot{u}_{\tau}=0 \\
\mu_{d}=0.4 & \text { if } & \dot{u}_{\tau}<0
\end{array}\right.
$$

For slip and stick different equations of motion(EOM) are set up. The EOM of the stick condition are presented in equations 4 to 6 . The system is linearized for

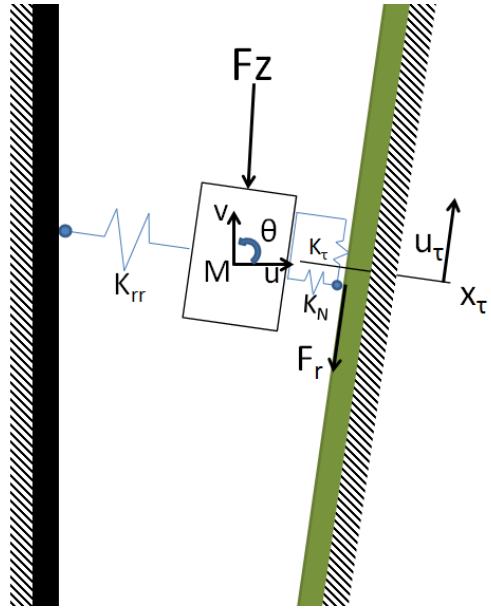

Figure 4: Mass-spring-friction oscillator

small angles, meaning that $\sin (\theta)=\theta, \cos (\theta)=1$ and the multiplication of $\theta$ with another displacements is $\ll 1$ and become negligible. The EOMs of slip are equal to the stick except that the tangential spring force is replaced by the friction force.

$$
\begin{aligned}
M \ddot{u}= & -\int_{0}^{L} K_{r r} u d X-\int_{0}^{L} K_{r r}\left(\frac{L}{2}-X\right) \sin (\theta) d X \\
& -K_{N} u_{N} \cos (\alpha)-K_{\tau}\left(u_{\tau}-x_{\tau}\right) \sin (\alpha) \\
M \ddot{v} & =K_{N} u_{N} \sin (\alpha)-K_{\tau}\left(u_{\tau}-x_{\tau}\right) \cos (\alpha)-F_{z}
\end{aligned}
$$

The normal and tangential displacements $U_{N}$ and $U_{\tau}$ are given by equations 7 and 8 and can be obtained with geometric relations and assuming small $\theta$ :

$$
\begin{aligned}
U_{N} & =u \cos (\alpha)-v \sin (\alpha)+\theta \frac{L}{2} \cos (\alpha) \\
U_{\tau} & =u \sin (\alpha)+v \cos (\alpha)+\theta \frac{L}{2} \sin (\alpha)
\end{aligned}
$$


To determine if slip or stick occurs, first the EOM of the stick situation are solved and the following condition is checked:

$$
\begin{array}{llll}
\text { Stick }: & \dot{u}_{\tau}=0 & \text { and } & F_{\tau} \leq \mu_{s} N \\
\text { Slip }: & u_{\tau} \neq 0 & \text { or } & F_{\tau} \geq \mu_{s} N \\
& & & \\
\text { With }: & F_{\tau}=K_{\tau} \cdot u_{\tau} &
\end{array}
$$

\subsection{Settlement under own weight}

The settlement under own weight is obtained using the dimensions of the monopile design of the Prinses Amelia wind farm, located $20 \mathrm{~km}$ off the coast of ljmuiden in the Netherlands. The design parameters of the monopile and of upper and lower part of the the slip joint are given in table 1 . The contact stiffnesses $K_{\tau}$ and $K_{N}$, and the radial stiffness $K_{r r}$ of the upper cone are calculated using the dimensions of table 1 and are presented in table 2 . The boundary condition for the contact stiffnesses are derived assuming that the upper end of the lower cone is free and that its lower end is clamped.

Table 1: Design parameters of the slip joint case study

\begin{tabular}{c|c|c|c} 
& Lower cone & Upper cone & Unit \\
\hline \hline Length & $L_{L c}=8$ & $L_{U c}=6$ & $\mathrm{~m}$ \\
Cone angle & $\alpha=2.5$ & $\beta=1.5$ & $\circ$ \\
Radius & $R_{L c}=2.0$ & $R_{U c}=1.74$ & $\mathrm{~m}$ \\
Wall thickness & 50 & 50 & $\mathrm{~mm}$ \\
Mass & & 27661 & $\mathrm{Kg}$
\end{tabular}

Table 2: Design parameters of the slip joint case study

\begin{tabular}{c|c|c} 
Parameter & Value & Unit \\
\hline \hline$E$ & $2.1 \mathrm{E} 11$ & $\mathrm{~N} / \mathrm{m} 2$ \\
$\nu$ & 0.33 & \\
$K_{\tau}$ & $0.42 \mathrm{E} 11$ & $\mathrm{~N} / \mathrm{m}$ \\
$K_{N}$ & $3.55 \mathrm{E} 10$ & $\mathrm{~N} / \mathrm{m}$ \\
$K_{r r}$ & $1.89 \mathrm{E} 11$ & $\mathrm{~N} / \mathrm{m}$
\end{tabular}

The desired overlap length, $L_{\text {overlap }}$, is assumed similar as for grouted connections, i.e. $1.5 D_{M P}$, where $D_{M P}$ is the diameter of the monopile. The distance that the upper cone must slip to attain full contact, designated $L_{\text {slip }}$, is dependent on the difference of the cone angles and the length of the upper cone as shown in figure 5.From figure 5 it can be seen that for a given set of initial cone angles, full contact can be ensured by a proper choice of diameter of the upper cone. The slip distance $L_{\text {slip }}$ and radius of the upper cone $R_{U c}$ are calculated as:

$$
\begin{aligned}
& L_{\text {slip }}=L_{\text {overlap }}-\frac{\sin (\alpha)}{L_{U c} \sin (\beta)} \\
& R_{U c}=.5 \cdot D_{M P}-L_{\text {slip }} \sin (\alpha)
\end{aligned}
$$

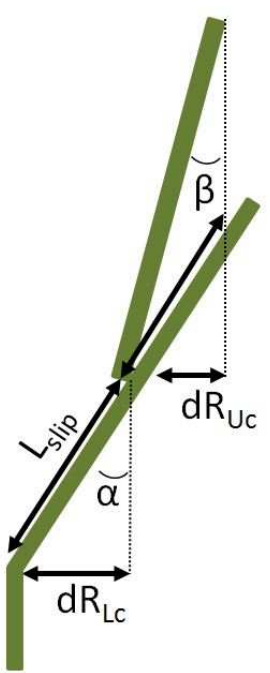

Figure 5: Slip distance clarification

The tangential displacement $U_{\tau}$ of the upper cone is plotted in figure 6 for the situation where the upper cone has no velocity or acceleration at the time of first contact. It can be seen that under the self weight only, very small slip distances are obtained and that this slip distance if is not satisfactory.

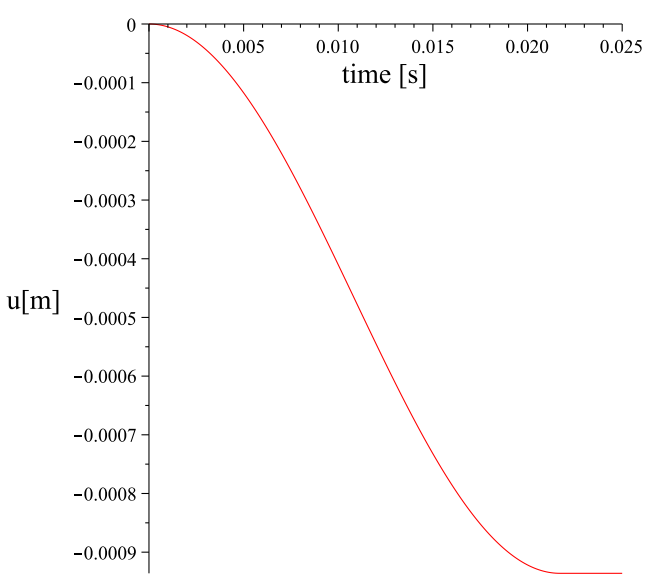

Figure 6: Tangential displacement under self weight 
Installation with an initial velocity The slipjoints onshore were installed by dropping them from approximately 10 $\mathrm{cm}$ high [3]. If this method is considered for the case given in table 1 this would lead to a velocity of about $1.4 \mathrm{~m} / \mathrm{s}$ on time of contact. The slip development due to this initial velocity is shown in figure 7 . The figure shows that this $10 \mathrm{~cm}$ drop will not lead to the desired overlap length for the considered geometry and stiffnesses. More research on the installation method and the sensitivity of the parameters on the settlement is therefore desired.

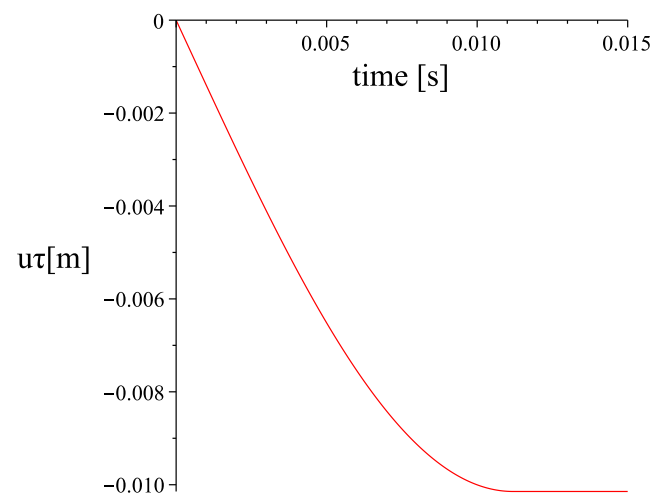

Figure 7: Tangential displacement under self weight with initial velocity $1.4 \mathrm{~m} / \mathrm{s}$

\subsection{Sensitivity of the settlement to cone angles and friction coefficient}

To investigate the sensitivity of the settlement to different cone angles and friction coefficients, the five cases listed in table 3 are considered. Case 1 is equal to the case described in table 1 with no initial velocity. Cases two and three consider the situation where, respectively, angle $\beta$ approaches angle $\alpha$ and the two cones are almost vertical. Cases four and and five consider the situation where the two cones are almost vertical in combination with lower dynamic friction coefficients. No initial velocity is taken into account for these five cases.

Table 3: Cases for the sensitivity of the slip joint settlement

\begin{tabular}{llccccc} 
Case & $\begin{array}{c}\alpha / \beta \\
{\left[{ }^{\circ}\right]}\end{array}$ & $\begin{array}{c}K_{N} \\
E 10[\mathrm{~N} / \mathrm{m}]\end{array}$ & $\begin{array}{c}K_{\tau} \\
E 11[\mathrm{~N} / \mathrm{m}]\end{array}$ & $\begin{array}{c}R_{U c} \\
{[\mathrm{~m}]}\end{array}$ & $\begin{array}{c}L_{\text {slip }} \\
{[\mathrm{m}]}\end{array}$ & $\begin{array}{c}\mu_{d} \\
{[-]}\end{array}$ \\
\hline \hline 1 & $2.5 / 1.5$ & 3.55 & 0.42 & 1.89 & 2.40 & 0.4 \\
2 & $2.5 / 2.4$ & 8.30 & 8.11 & 1.98 & 0.24 & 0.4 \\
3 & $1.1 / 1$ & 3.96 & 2.65 & 1.99 & 0.54 & 0.4 \\
4 & $1.1 / 1$ & 3.96 & 2.65 & 1.99 & 0.54 & 0.3 \\
5 & $1.1 / 1$ & 3.96 & 2.65 & 1.99 & 0.54 & 0.2 \\
\hline \hline
\end{tabular}

In figure 8 the tangential displacement $u_{\tau}$ of cases 1 to 3 are presented. Comparing the slip of case 1 with case 2 , we can conclude that the increase of the angle $\beta$ has two effects. First it causes the position of contact to be closer to the stiff lower boundary. Secondly, this position of contact leads to larger contact stiffnesses and shorter slip distances. For case 3,i.e. smaller angles of $\alpha$ and $\beta$, the contact stiffness slightly increase compared to case 1 and the steeper slope enables a larger slip distance as can be seen in figure 8 .

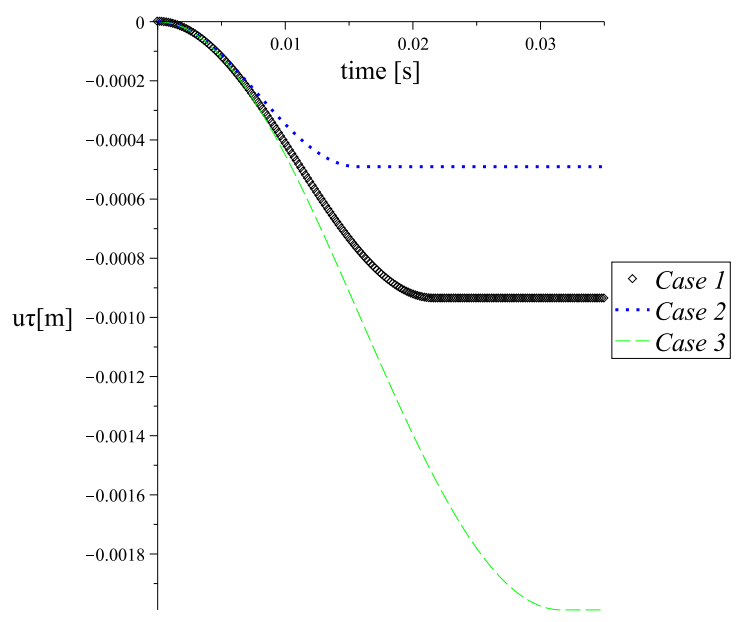

Figure 8: Tangential displacement under self weight for cases 1 to 3 of table 3

In figure 9 the results of cases 3 to 5 are presented. It can be seen that for lower friction coefficients the slip distance increases.

On the basis of these results it can be concluded that the tangential displacement caused by the self weight of the slipjoint is insufficient to come to a desired contact overlap for small angles, small angles differences and low friction coefficients. For a slipjoint, installation under self weight is therefore considered to be undesirable and insufficient. Future research in the installation of the slipjoint will therefore include harmonic loads for the installation.

\section{FE modeling}

In the second part of this contribution, the static capacities (axial and bending/shear) of the slipjoint is analyzed using the FE program ANSYS. To determine their relative importance, the effect on the results of the following parameters is analyzed:

- the cone angle

- the friction coefficient $\mu$

- the size of the contact area (overlap)

- the diameter $\mathrm{D}$ 


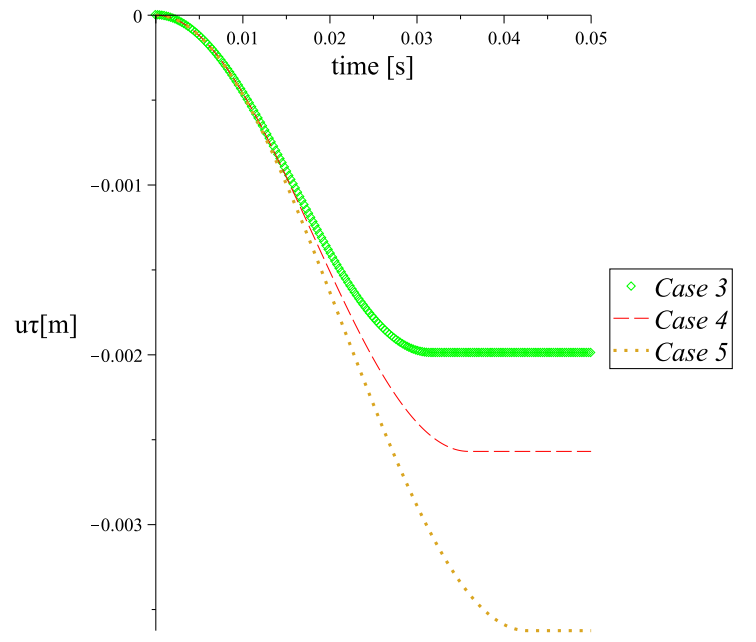

Figure 9: Tangential displacement under self weight for cases 3 to 5 of table 3

- the wall thickness $t$.

The FE analyses also serve to indicate other important quantities of interest, e.g. contact pressure distributions, settlement, etc. Based on the results, some initial design recommendations are made.

\subsection{Model description}

Figure 10 shows the two parts of a representative slipjoint as these were modelled in ANSYS. The walls of the monopile and transition piece were modelled using 8 -node, $2^{\text {nd }}$-order SHELL281 elements having 6 degrees of freedom (DOF) at each node. The figure also shows the applied boundary conditions (clamped at the base). The Young's modulus and Poisson's ratio of steel is taken as $207 \mathrm{GPa}$ and 0.3 , respectively.

To apply loads to the top of this assembly, a master node was defined at the top and centre of the transition piece and connected to its walls using rigid beams. These beams were modelled using the multipoint constraint element MPC184, and are also indicated in figure $10 \mathrm{~b}$.

To model the contact between the monopile and the transition piece a surface-to-surface contact model was set up by overlaying the conical parts of the assembly with 8-node contact elements (CONTA174). Since the contact can be assumed to be symmetric, i.e. neither of the surfaces can clearly be designated a target surface, target elements TARGE170 were also overlayed on both conical parts, and ANSYS was asked to internally select a contact-target designation at the solution stage $(\operatorname{KEYOPT}(8=2))$. To exclude possible inaccuracies in the contact stresses due to the use of faceted surfaces in stead of the true curved geometry, a cylindrical geometry cor-

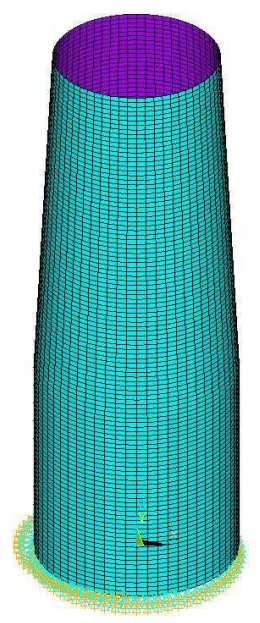

(a) Monopile

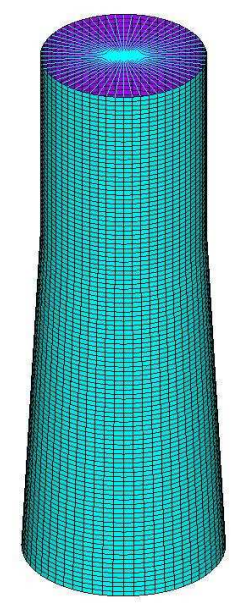

(b) Transition piece
Figure 10: Model of the slipjoint parts belonging to the a) monopile and b) transition piece.

rection was applied to both contact surfaces before solving. A basic Coulomb model, with zero cohesion sliding resistance, was used to model the friction. Given the large influence of the initial contact status on convergence, initial contact gaps were closed $(\mathrm{KEYOPT}(5)=1)$.

\subsection{Results}

The response of the slipjoint to the following two static load cases was analyzed:

Load case 1 (LC1): Axial load of $2 \mathrm{MN}$.

Load case 2 (LC2): Bending moment of $60 \mathrm{MNm}$ and shear force of $1 \mathrm{MN}$.

All forces are applied to the master node as described above in section 3.1. Table 4 shows some of the results obtained for slipjoints having different geometrical and frictional properties, with $s, \sigma_{\mathrm{vm}}^{\max }$ and $p_{\max }$ referring to the settlement (LC1), maximum Von Mises stress (LC2), and maximum contact pressure (LC2), respectively. The latter two quantities are not reported for LC1, their being significantly less than those obtained for LC2. A representative contour plot of the Von Mises stresses for LC1 is shown in figure 11 and 12. The values of the stresses reported in the figure were obtained using property set 5 in table 4.

The slipjoint's response to a static bending moment and shear force (LC2) is considered next. Typical displacement contours and deformations (scaled) for this load case are shown in figure 13(a). 
Table 4: Settlement (LC1), maximum Von Mises stress $\sigma_{\mathrm{vm}}^{\max }$ (LC2) maximum contact pressure $p_{\max }$ (LC2) and response type (LC2) for slipjoints having different geometrical and frictional properties.

LC1

LC2

\begin{tabular}{cccccccccc}
\hline $\begin{array}{c}\text { Set } \\
\text { nr. }\end{array}$ & $\begin{array}{c}\text { angle } \\
{\left[{ }^{\circ}\right]}\end{array}$ & $\begin{array}{c}\mathrm{D} \\
{[\mathrm{m}]}\end{array}$ & $\mu$ & overlap & $\begin{array}{c}t \\
{[\mathrm{~mm}]}\end{array}$ & $\begin{array}{c}s \\
{[\mathrm{~mm}]}\end{array}$ & $\begin{array}{c}\sigma_{\mathrm{vm}}^{\max }[\mathrm{MPa}] \\
{\left[\begin{array}{c}{ }^{2} \\
{[\mathrm{Mpa}]}\end{array}\right.}\end{array}$ & $\begin{array}{c}\text { response } \\
\text { type }\end{array}$ \\
\hline 1 & 3 & 4 & 0.2 & $1.2 \mathrm{D}$ & 40 & 2.51 & 366.21 & 12.16 & $\mathrm{a}$ \\
2 & 3 & 4 & 0.4 & $1.2 \mathrm{D}$ & 40 & 1.54 & 287.94 & 9.61 & $\mathrm{a}$ \\
3 & 3 & 4 & 0.8 & $1.2 \mathrm{D}$ & 40 & 0.97 & 227.43 & 6.94 & $\mathrm{~b}$ \\
4 & 3 & 4 & 0.2 & $1.5 \mathrm{D}$ & 40 & 2,08 & 266.71 & 8.93 & $\mathrm{a}$ \\
5 & 3 & 4 & 0.4 & $1.5 \mathrm{D}$ & 40 & 1.31 & 246.15 & 7.30 & $\mathrm{~b}$ \\
6 & 3 & 4 & 0.8 & $1.5 \mathrm{D}$ & 40 & 0.86 & 245.3 & 5.52 & $\mathrm{~b}$ \\
7 & 3 & 4 & 0.2 & $1.8 \mathrm{D}$ & 40 & 1.78 & 266.44 & 7.50 & $\mathrm{~b}$ \\
8 & 3 & 4 & 0.4 & $1.8 \mathrm{D}$ & 40 & 1.15 & 265.69 & 6.34 & $\mathrm{~b}$ \\
9 & 3 & 4 & 0.8 & $1.8 \mathrm{D}$ & 40 & 0.79 & 264.60 & 4.98 & $\mathrm{~b}$ \\
10 & 1 & 4 & 0.2 & $1.2 \mathrm{D}$ & 40 & 8.49 & 331.77 & 10.60 & $\mathrm{a}$ \\
11 & 1 & 4 & 0.4 & $1.2 \mathrm{D}$ & 40 & 4.58 & 263.97 & 8.46 & $\mathrm{a}$ \\
12 & 1 & 4 & 0.8 & $1.2 \mathrm{D}$ & 40 & 2.52 & 190.05 & 6.09 & $\mathrm{a}$ \\
13 & 1 & 4 & 0.2 & $1.5 \mathrm{D}$ & 40 & - & 234.25 & 7.28 & $\mathrm{a}$ \\
14 & 1 & 4 & 0.4 & $1.5 \mathrm{D}$ & 40 & 3.76 & 191.90 & 5.97 & $\mathrm{a}$ \\
15 & 1 & 4 & 0.8 & $1.5 \mathrm{D}$ & 40 & 2.12 & 164.45 & 4.48 & $\mathrm{c}$ \\
16 & 1 & 4 & 0.2 & $1.8 \mathrm{D}$ & 40 & 5.78 & 185.37 & 5.74 & $\mathrm{a}$ \\
17 & 1 & 4 & 0.4 & $1.8 \mathrm{D}$ & 40 & 3.19 & 167.46 & 4.83 & $\mathrm{C}$ \\
18 & 1 & 4 & 0.8 & $1.8 \mathrm{D}$ & 40 & 1.85 & 167.63 & 3.75 & $\mathrm{c}$ \\
19 & 3 & 5 & 0.2 & $1.5 \mathrm{D}$ & 40 & 2.05 & 173 & 4.99 & $\mathrm{a}$ \\
20 & 3 & 5 & 0.4 & $1.5 \mathrm{D}$ & 40 & 1.28 & 157.29 & 4.08 & $\mathrm{~b}$ \\
21 & 3 & 5 & 0.8 & $1.5 \mathrm{D}$ & 40 & 0.83 & 156.75 & 3.08 & $\mathrm{~b}$ \\
22 & 3 & 4 & 0.2 & $1.5 \mathrm{D}$ & 60 & 1.41 & 171.94 & 8.29 & $\mathrm{a}$ \\
23 & 3 & 4 & 0.4 & $1.5 \mathrm{D}$ & 60 & 0.88 & 155.59 & 6.79 & $\mathrm{~b}$ \\
24 & 3 & 4 & 0.8 & $1.5 \mathrm{D}$ & 60 & 0.58 & 154.86 & 5.13 & $\mathrm{~b}$ \\
\hline & & & & & & & & &
\end{tabular}

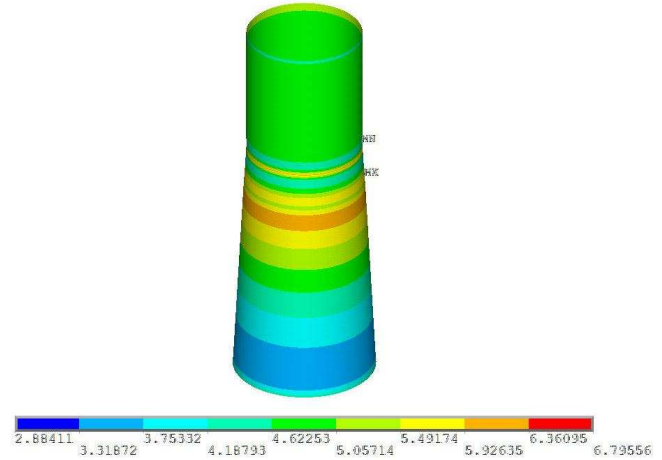

Figure 11: Von Mises stress contours for the transition piece when subjected to LC1. Stresses are in MPa and were obtained using property set number 5 (cf. table 4). Deformations are scaled.

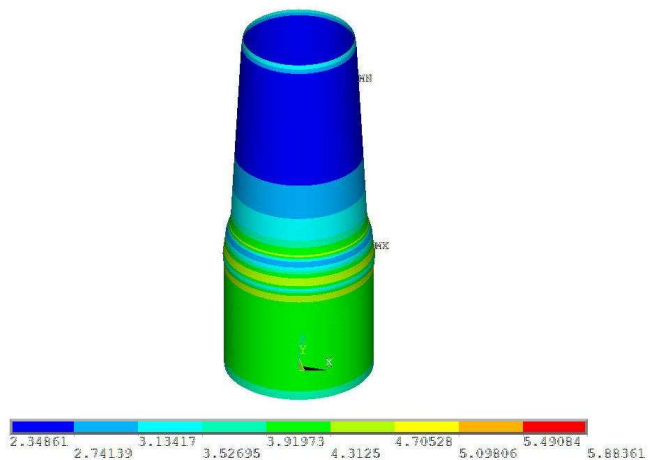

Figure 12: on Mises stress contours for the monopile when subjected to LC1. Stresses are in MPa and were obtained using property set number 5 (cf. table 4). Deformations are scaled.

For the 24 cases specified in table 4, 3 different types of re- each of these 3 cases are plotted in figure 14(a). In the first sponse could be identified. The Von Mises stress contours for case, the maximum stress occurs at the top of the monopile. 
This situation is encountered in almost all cases when the value of the friction coefficient is low $(\mu=0.2)$ as well as for overlaps of 1.2D (independent of the friction coefficient).

The second type of stress pattern, figure 14(b), corresponds to situations where the maximum stress occurs in the top part of the joint at the transition from a conical to a cylindrical section. This type of response occurs when the value of the friction coefficient is high $(\mu=0.8)$, except in the case of small cone angles $\left(1^{\circ}\right)$ and overlaps (1.2D).

Finally, figure 14(c) represents a situation in which the maximum stress is found at the bottom of the modelled part of the monopile, close to the support, indicating a succesfull transferral of the loads from the transition piece to the monopile. For the slipjoints listed in table 4, this situation is only encountered for cone angles of $1^{\circ}$ with large overlaps (1.8D) and friction coefficients $\mu \geq 0.4$, or with smaller overlaps (1.5D) but even more friction $\mu \geq 0.8$.

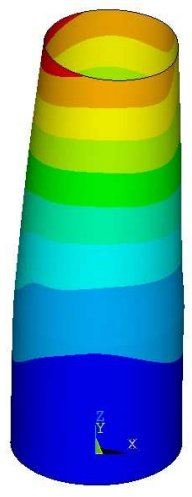

(a) Bottom cone

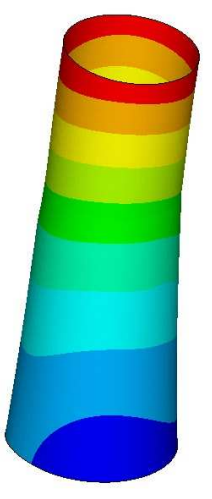

(b) Top cone
Figure 13: Typical displacement contours and deformations for the a) bottom and b) top cone when subjected to LC2. Deformations are scaled.

Typical contact pressure distribution and contact status contours for LC2 are shown in figures 15- 17. Given the zero cohesion friction model, the contact status keeps alternating between 'open' and 'sliding', with the two 'open' contact areas on the respective sides of the cone breaking through and meeting each other for large overlaps and high friction coefficients - cf. figures 16 and 17.

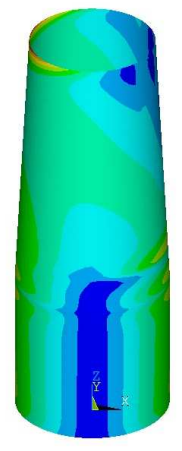

(a) Bottom cone

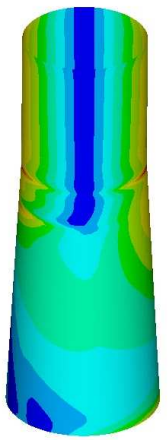

(b) Top cone

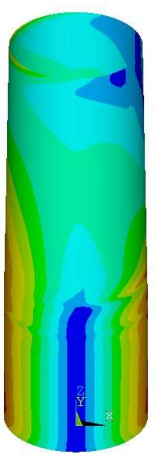

(c) Bottom cone

Figure 14: Three different types of response of the slipjoint when simultaneously subjected to a static shear force and moment (LC2).

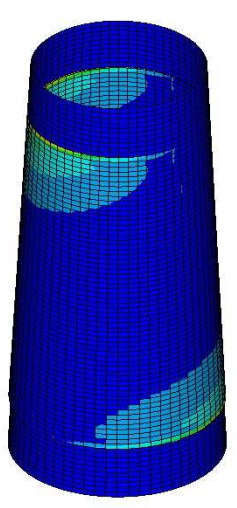

Figure 15: Contact pressure for LC2 and property set 5 in table 4

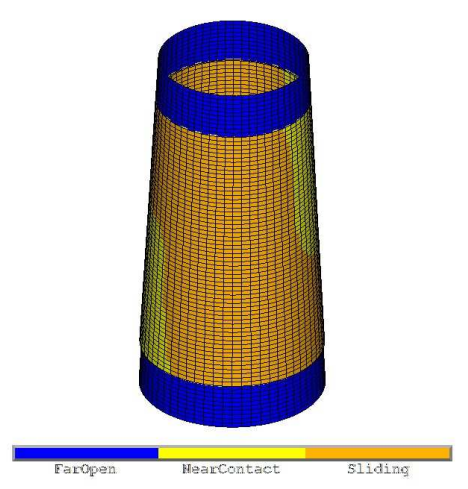

Figure 16: Contact status for LC2 and property set 5 in table 


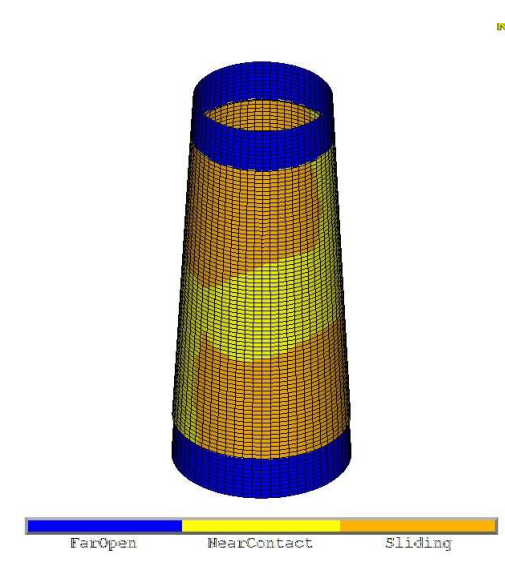

Figure 17: Contact status for LC2 and property set 9 in table 4

\section{Conclusions and outlook}

Two different aspects of the development of a slip joint for offshore applications were considered, namely a) a simplified dynamic analysis of its installation under own weight and b) a determination of its static capacities (axial and bending) in the in-place situation by means of a FE model. From the dynamic installation analysis it is concluded that even for small initial cone angles, small angle differences between the top and bottom cone, and low friction coefficients, the tangential displacement caused by the self weight is insufficient to come to a desired contact overlap. Future research will therefore focus on the use of applied harmonic forces as well as the influence of additional weight on the installation. Secondly, during the contact analyses with the FE model, small cone angles and large overlaps were identified as most conducive to a succesfull transferral of the loads from the transition piece to the monopile. Given the uncertainty on the friction coefficient, it is then also recommended to use a cone angle of $1^{\circ}$ and preferably an overlap $>1.5 \mathrm{D}$. For final design recommendations, however, a complementary dynamic assesment is necessary.

\section{References}

[1] Det Norske Veritas, Joint Industry Project Summary Report From The JIP on the Capacity of Grouted Connections in Offshore Wind Turbine Structures Technical Report No: 2010-1053, rev. 05

[2] Det Norske Veritas,DNV-OS-J101 Design of offshore wind turbine structures,November 2007(adjusted November 2009)

[3] van der Tempel \& Lutje Schipholt, The Slip-Joint Connection, Alternative connection between pile and tower, DOWEC report-F1W2-JvdT-03-093/01-P, 2003

[4] William S. Slaughter, The linearized Theory of Elasticity, Ch.7, Birkhäuser Boston, 2002

[5] H. Hong \& C. Lui, Coulomb friction oscillator: Modeling and responses to harmonic loads and base excitations, Journal of Sound and Vibrations 229,2000

[6] J.P. Den Hartog, Forced vibrations with combined viscous and Coulomb damping,Transactions of American Society of Mechanical Engineers 53, 1931

[7] K. Popp, Non-Smooth Mechanical Systems - An Overview, Forchung im Engenieurwesen 64, 223-229, Springer, 1998

[8] Werner Soedel, Vibrations of Shells and Plates, Ch. 3.2, Marcel Dekker, 2004 